\title{
Simulation of the scrape-off layer region of tokamak devices
}

\author{
Paolo Ricci† \\ École Polytechnique Fédérale de Lausanne (EPFL), \\ Centre de Recherches en Physique des Plasmas (CRPP), CH-1015 Lausanne, Switzerland \\ (Received 15 September 2014; revised 18 November 2014; accepted 19 November 2014; \\ first published online 12 February 2015)
}

Understanding the key processes occurring in the tokamak scrape-off layer (SOL) is becoming of the outmost importance while we enter the ITER era and we move towards the conception of future fusion reactors. By controlling the heat exhaust, by playing an important role in determining the overall plasma confinement, and by regulating the impurity level in tokamak core, the dynamics of the fusion fuel in the SOL is, in fact, related to some of the most crucial issues that the fusion program is facing today. Because of the limited diagnostic access and in view of predicting the SOL dynamics in future devices, simulations are becoming crucial to address the physics of this region. The present paper, which summarizes the lecture on SOL simulations that was given during the 7th ITER international school (August 25-29, 2014, Aix-en-Provence, France), provides a brief overview of the simulation approaches to the SOL dynamics. First, disentangling the complexity of the system, the key physics processes occurring in the SOL are described. Then, the different simulation approaches to the SOL dynamics are presented, from first-principles kinetic and fluid models, to the phenomenological analysis.

\section{Introduction}

It is often the case that the boundary conditions strongly influence, or even determine, the dynamics of a system. While in the early days of fusion research the edge region was not of primary interest, as the main focus was on the core region where fusion reactions can take place, it was soon discovered that the edge was going to require considerable attention. Early devices tended to be dominated by radiations from impurities, originated from the plasma-surface interaction. The radiative cooling of these impurities was so strong that the core temperature needed to achieve fusion was far from being achieved. Nowadays, it turns out that one of the greatest uncertainties in the success of ITER and future fusion reactors is related to the dynamics of the plasma fusion fuel in the SOL, the most external plasma region surrounding the tokamak core, where magnetic field lines are open and terminate on the vessel of the device (ITER Physics Expert Group on Divertor et al. 1999; Stangeby 2000; Loarte et al. 2007).

The plasma behavior in this region governs the overall confinement properties of the device. This is critically important since conditions for self-sustained fusion reactions can be achieved only in sufficiently well-confined, high-temperature, and high-density plasmas. SOL plasma phenomena also regulate the impurity dynamics 
and the level of fusion ashes, which can dilute the fusion fuel, stopping the reactions. Moreover, the SOL dynamics determines the heat load to the tokamak vessel walls a showstopper for the whole fusion program if the material requirements cannot be met (Eich et al. 2011; Zohm et al. 2013).

Approaching the study of SOL physics is particularly challenging. First, transport of density and heat in this region is governed by highly nonlinear turbulent processes, occurring on multiple spatiotemporal scales, driven by different energy sources, mainly the inhomogeneity of plasma pressure profiles and confining magnetic field, and with a number of self-regulating mechanisms (Yagi et al. 2008). Second, the magnetic configuration is particularly complex. As a consequence, typical coordinate systems used for core plasma simulations are found to be singular in the SOL. Third, typical assumptions used in the study of turbulence in the tokamak core (e.g. small turbulent fluctuations) are not valid in the SOL. In fact, the intermittent character of SOL transport, dominated by large fluctuations, comparable to the equilibrium quantities, does not allow separation between transport events and system size. Even the meaning of equilibrium profiles in the SOL is questionable. Fourth, the plasma properties across the SOL change significantly, e.g. in terms of collisionality. Moreover, close to the solid wall, at the sheath, plasma is not neutral and plasma particle motion cannot be described in terms of their drifts, as it is typically the case in the rest of the SOL. Finally, the dynamics of neutral atoms is found to play a key role in the SOL dynamics, with a number of collisional and atomic physics processes taking place (Stangeby 2000).

Simulations of the plasma dynamics in the SOL region constitute an invaluable tool for the understanding of the basic physics processes at play, and are acquiring an increasing role in the predictions of the SOL dynamics in future fusion reactors. With respect to the core region, the SOL region is accessible for diagnostics with probes, at least in smaller devices. The recent significant development of these probes (see e.g. LaBombard et al. (2014)) provides constraining measurement for the validation of the simulation results. First-principles simulations of the SOL are carried out using both kinetic and fluid models. Usually considering a one-dimensional configuration, the kinetic models allow the description of the self-consistent interaction between plasma, neutral atoms, and solid wall (Tskhakaya 2012). These simulations constitute an ideal tool to identify the most important interactions occurring between neutral atoms and plasma, to evaluate the parallel transport coefficients, and to identify the boundary conditions to be applied at the plasma-wall transition. All those elements are essential in the development of three-dimensional SOL simulation codes that are typically based on fluid equations.

The fluid-like equations that are generally considered appropriate to investigate plasma turbulence in the SOL were first developed by Braginskii (1965). More precisely, based on the Braginskii equations, a number of reduced models more suited for computational treatment have been deduced (see, e.g. Zeiler et al. (1997)), in some cases taking into account effects neglected in the original Braginskii equations (Ribeiro and Scott 2005). A number of codes have then been developed based on these reduced models, constituting ideal flexible tools to perform the large parameter scans of simulations needed to achieve a deep understanding of the SOL turbulence. The coupling of the plasma fluid equations with models describing neutral particle dynamics and sheath physics is now being approached by some groups.

While first-principles simulations are rapidly progressing and are shedding light on the basic physics processes at play in the SOL, the tools of reference for the design of ITER and future reactors were and remain simulation codes based on a 
phenomenological approach (Kukushkin et al. 2011). By modeling the perpendicular transport as a diffusive process, the SOL profile can be described in realistic tokamak geometry, taking into account the interaction with the neutral atoms and the plasma. The phenomenological models can be used in interpretative mode, to evaluate the free parameters, in particular the diffusion coefficients, that best fit the experimental data, and in predictive modes, extrapolating the diffusion coefficients to the regime of interest.

The present paper, which summarizes the lecture on SOL simulations that was given at the 7th ITER international school (August 25-29, 2014, Aix-en-Provence, France), is devoted to the description of the key elements that play a role in the SOL and of the typical models that are used for its simulations. It is structured as follows. In Sec. 2, the main elements of the SOL physics are introduced. A description of a complete kinetic model for the SOL is given in Sec. 3. Section 4 describes the fluid modeling of the SOL. A short summary of the main elements involved in the phenomenological approach are reported in Sec. 5. We finally draw our conclusions in Sec. 6.

\section{Key elements of SOL physics}

In a fusion reactor, the edge should be designed in order to (i) fuel the plasma, controlling the plasma density and fusion power, (ii) exhaust the He ashes, (iii) ensure a level of impurities, as well as of neutral atoms, in the core, low enough so that the machine performance are not degraded, and (iv) exhaust the heating power, with acceptable loads on the plasma facing components, i.e. $10 \mathrm{MW} \mathrm{m}^{-2}$ for present materials. To perform these tasks efficiently, complex magnetic configurations have to be considered, with a number of interconnected physical processes being involved. Here, we briefly recall the key physical processes that play a role in the SOL and that have led to the design of the current configurations. The discussion is based on the complete overview of the key elements of SOL physics presented in Stangeby (2000).

In a tokamak, the set of nested toroidal flux surfaces created to confine the plasma has to be interfaced with the solid wall of the device. The simplest realization of this interface is constituted by a solid wall that follows the most external flux surface. It is extremely challenging to realize such a configuration as small errors in the localization of the plasma current, for example, can move the flux surfaces unpredictably. The most external surfaces can, therefore, get in contact with the vessel and the magnetic field lines lying on those surfaces be broken, ending on the solid wall. Charged particles move freely along the magnetic field lines and can easily reach the vessel. When electrons and ions strike a solid surface, they tend to stick to it, until they recombine and are released in the form of neutrals. We note that this process, called recycling, leads to a flux of neutrals, whose ionization accounts for the main plasma source in tokamak. Therefore, the solid surface acts as sink of plasma (and a source of neutral), causing the rapid removal of ions and electrons at a speed that is, approximatively, the sound speed. As a consequence, while the plasma slowly diffuses across the magnetic flux surfaces, it rapidly flows along the magnetic field lines, and it is swept out from the system. It follows that the plasma density decreases to a negligible value, over a thin radial layer, and that the heat and particle are dissipated on a localized region of the vessel. This might lead to a localized overheating of the wall, its erosion, and the production of impurities that cause a loss of fusion performances.

In order to localize and better control the interaction of the plasma with the vessel of the device, limiters have been inserted on the first wall. Limiters can be, for example, 
an annulus of solid material inserted at one or more toroidal locations, or a rail that runs toroidally over the full device. Limiters well define the SOL, the region where the magnetic field lines are open, separated from the closed flux surface region by the last closed flux surface. While limited tokamak are still operational, in state-of-the-art tokamak the divertor configuration is preferred. These can be obtained by using an external conductor carrying a current in the direction of the plasma current. In the poloidal plane, the field lines make a figure-of-eight shape, with an $\mathrm{X}$ point. Beside the fact that the high-confinement mode is accessed more easily in diverted than in limited configurations, there are a number of other reasons to prefer divertors to limiters, despite the fact that the divertors do not allow the optimal use of the plasma volume. In fact, by accessing a wider operational space, diverted configurations enable a better handling of the heat exhaust and limit the production of the impurities and their transport to the core.

In limited configurations, being emitted close to the last closed flux surface, the recycling neutrals can easily penetrate in the core region where they are ionized. By outflowing from the core, these recycled particles give a significant parallel plasma flow and hence a significant parallel heat convection along the field lines of the SOL. It follows that the plasma temperature remains constant along these field lines, up to their ends on the vessel, with no significant temperature drop. In this regime, called convection limited or sheath limited regime, the only way to have a low temperature at the vessel wall, favorable for reduced impurity production, is to have low temperature at the last closed flux surface. This is detrimental for tokamak performances and can, moreover, be achieved only at high density, giving rise to density limit problem.

Diverted tokamak can be operated in the sheath limited regime. However, the configuration allows some flexibility and the access to other operational regimes. In fact, if the ionization mean free path is made short, by increasing the plasma density, ionization might occur only in the SOL, close to the source of the recycling neutrals, i.e. the divertor plates. Heat convection is reduced, conduction becomes the main transport channel, and the plasma temperature drops at the divertor plates while the temperature of the plasma in contact with the core remains high. The high neutral density close to the divertor plate causes a neutral frictional drag that slows the ions while they approach the solid wall. Lower temperature and neutral friction drag lead to a reduced impurity production. As plasma pressure is about constant along a field line, the plasma density increases close to the divertor plates, as well as the flux of particles and, therefore, the recycling. This is why this regime is called high recycling or conduction limited. We note that the high neutral pressure facilitates the pumping, and therefore the removal of fusion ashes from the plasma.

As the intensity of the recycling increases, the temperature decreases. At low temperature, a large number of excitations occur before ionization and the radiative losses increase. Moreover, if one considers the low $\mathrm{Z}$ elements, the radiation is higher at lower temperature. The increase of radiation leads to a reduced heat load on the plasma wetted area; this can also be enhanced by introducing impurities, with no major concerns about polluting the core plasma. In addition, at low temperature, neutral frictional drag and recombination may become strong, which reduce the plasma density and the temperature furthermore (recombination contributes to the radiated process). The consequence is a reduced flux to the divertor, reduced impurity production, and reduced heat load. This is the detachment regime, which can also be achieved in limited configurations by injecting impurities, such as neon, and by creating a radiative mantle. However, the contamination of the main plasma might be a concern in limited configurations. 
This phenomenological overview of the SOL operating regimes highlights the key features that one has to consider while approaching the simulation of the SOL dynamics. The SOL plasma model should describe the plasma transport along and across the magnetic field lines. At the same time, a model able to capture the interaction of the plasma with the neutrals and with the solid surface is necessary. Therefore the plasma equations, provided with a good set of boundary conditions, should be advanced self-consistently with a model describing the neutral dynamics.

\section{Kinetic description of the SOL dynamics}

A fairly complete model of the SOL dynamics is based on a kinetic description of the electrons, the ions, and the neutral atoms. Assuming that there is only one neutral species, which undergoes ionization, charge exchange with the ion population, and recombination processes (the description can be easily generalized to multiple neutral species, considering different excitation states, taking into account molecular dissociation processes, as well as the radiation production associated with these processes), the neutral atom distribution function $f_{n}\left(\mathbf{x}, \mathbf{v}_{n}, t\right)$ satisfies the following kinetic equations:

$$
\begin{aligned}
& \frac{\partial f_{n}\left(\mathbf{x}, \mathbf{v}_{n}, t\right)}{\partial t}+\mathbf{v}_{n} \cdot \frac{\partial f_{n}\left(\mathbf{x}, \mathbf{v}_{n}, t\right)}{\partial \mathbf{x}}=-f_{n}\left(\mathbf{x}, \mathbf{v}_{n}, t\right) \int \sigma_{i o n}\left(v_{e}\right) v_{e} f_{e}\left(\mathbf{x}, \mathbf{v}_{e}, t\right) d \mathbf{v}_{e} \\
& \quad-\int \sigma_{C X}\left(\mathbf{v}_{n}-\mathbf{v}_{i}\right)\left|\mathbf{v}_{n}-\mathbf{v}_{i}\right|\left[f_{n}\left(\mathbf{x}, \mathbf{v}_{n}, t\right) f_{i}\left(\mathbf{x}, \mathbf{v}_{i}, t\right)-f_{i}\left(\mathbf{x}, \mathbf{v}_{n}, t\right) f_{n}\left(\mathbf{x}, \mathbf{v}_{i}, t\right)\right] d \mathbf{v}_{i} \\
& \quad+f_{i}\left(\mathbf{x}, \mathbf{v}_{n}, t\right) \int \sigma_{r e c}\left(v_{e}\right) v_{e} f_{e}\left(\mathbf{x}, \mathbf{v}_{e}, t\right) d \mathbf{v}_{e},
\end{aligned}
$$

where $\sigma_{i o n}, \sigma_{C X}, \sigma_{r e c}$ are the ionization, charge exchange, and recombination cross sections, and we have assumed $v_{n}, v_{i} \ll v_{e}$. The ion kinetic equation reads as

$$
\begin{aligned}
& \frac{\partial f_{i}\left(\mathbf{x}, \mathbf{v}_{i}, t\right)}{\partial t}+\mathbf{v}_{i} \cdot \frac{\partial f_{i}\left(\mathbf{x}, \mathbf{v}_{i}, t\right)}{\partial \mathbf{x}}+\frac{q_{i}}{m_{i}}\left(\mathbf{E}+\frac{1}{c} \mathbf{v}_{i} \times \mathbf{B}\right) \cdot \frac{\partial f_{i}\left(\mathbf{x}, \mathbf{v}_{i}, t\right)}{\partial \mathbf{v}_{i}} \\
& =C\left(f_{i}, f_{i}\right)+C\left(f_{i}, f_{e}\right)+f_{n}\left(\mathbf{x}, \mathbf{v}_{i}, t\right) \int \sigma_{i o n}\left(v_{e}\right) v_{e} f_{e}\left(\mathbf{x}, \mathbf{v}_{e}, t\right) d \mathbf{v}_{e} \\
& \quad-\int \sigma_{C X}\left(\mathbf{v}_{n}-\mathbf{v}_{i}\right)\left|\mathbf{v}_{n}-\mathbf{v}_{i}\right|\left[f_{i}\left(\mathbf{x}, \mathbf{v}_{i}, t\right) f_{n}\left(\mathbf{x}, \mathbf{v}_{n}, t\right)-f_{n}\left(\mathbf{x}, \mathbf{v}_{i}, t\right) f_{i}\left(\mathbf{x}, \mathbf{v}_{n}, t\right)\right] d \mathbf{v}_{n} \\
& \quad-f_{i}\left(\mathbf{x}, \mathbf{v}_{i}, t\right) \int \sigma_{r e c}\left(v_{e}\right) v_{e} f_{e}\left(\mathbf{x}, \mathbf{v}_{e}, t\right) d \mathbf{v}_{e}
\end{aligned}
$$

where $C\left(f_{i}, f_{i}\right)$ and $C\left(f_{i}, f_{e}\right)$ represent the ion-ion and ion-electron Coulomb collisions, which cannot be neglected in the SOL due to the low plasma temperature. The kinetic equations for the electrons is

$$
\begin{aligned}
\frac{\partial f_{e}\left(\mathbf{x}, \mathbf{v}_{e}, t\right)}{\partial t}+\mathbf{v}_{e} \cdot \frac{\partial f_{e}\left(\mathbf{x}, \mathbf{v}_{e}, t\right)}{\partial \mathbf{x}}+\frac{q_{e}}{m_{e}}\left(\mathbf{E}+\frac{1}{c} \mathbf{v}_{e} \times \mathbf{B}\right) \cdot \frac{\partial f_{e}\left(\mathbf{x}, \mathbf{v}_{e}, t\right)}{\partial \mathbf{v}_{e}} \\
=C\left(f_{e}, f_{e}\right)+C\left(f_{e}, f_{i}\right)-n_{n} \sigma_{i o n}\left(v_{e}\right) v_{e} f_{e}\left(\mathbf{x}, \mathbf{v}_{e}, t\right) \\
\quad+2 n_{n} \int \sigma_{i o n}\left(v_{e}^{\prime}\right) \Phi_{i o n}\left(\mathbf{v}_{e}^{\prime}, \mathbf{v}_{e}\right) v_{e}^{\prime} f_{e}\left(\mathbf{x}, \mathbf{v}_{e}^{\prime}, t\right) d \mathbf{v}_{e}^{\prime}-n_{i} \sigma_{r e c}\left(v_{e}\right) v_{e} f_{e}\left(\mathbf{x}, \mathbf{v}_{e}, t\right) \\
\quad-n_{n} \sigma_{e l}\left(v_{e}\right) v_{e} f_{e}\left(\mathbf{x}, \mathbf{v}_{e}, t\right)+\frac{1}{4 \pi} n_{n} \int \sigma_{e l}\left(v_{e}\right) v_{e} f_{e}\left(\mathbf{x}, \mathbf{v}_{e}, t\right) d \boldsymbol{\Omega},
\end{aligned}
$$


where $\Phi_{i o n}\left(\mathbf{v}_{e}^{\prime}, \mathbf{v}_{e}\right)$ is the distribution function of the electrons resulting from the ionization process when the impacting electron has velocity $\mathbf{v}_{e}^{\prime}$ and it is such that $\int \Phi_{i o n}\left(\mathbf{v}_{e}^{\prime}, \mathbf{v}_{e}\right) d \mathbf{v}_{e}=1$. The $\sigma_{e l}$ represents the cross section of the electron-neutral elastic collisions.

In the simplest case of a totally absorbing wall, $\mathbf{x}=\mathbf{x}_{w}$, that instantaneously re-emits the absorbed ions as recycling neutrals, the boundary conditions read as $f_{i}\left(\mathbf{x}_{w}, \mathbf{v}, t\right)=0$ and $f_{e}\left(\mathbf{x}_{w}, \mathbf{v}, t\right)=0$ for all $\mathbf{v}$ such that $\mathbf{v} \cdot \mathbf{n}<0$ where $\mathbf{n}$ is the unitary vector normal to the boundary surface, pointing outwards. For the neutral atoms, one imposes that the recycling ions and the impacting neutrals are emitted from the surface according to the following distribution function $f_{n}\left(\mathbf{x}_{w}, \mathbf{v}, t\right) \propto \cos (\theta) \exp \left[-m v^{2} / 2 T_{w}\right]$ for $\mathbf{v} \cdot \mathbf{n}<0$, with the normalization constant defined by setting

$$
\int f_{n}\left(\mathbf{x}_{w}, \mathbf{v}, t\right) v_{\perp} d \mathbf{v}+\int f_{i}\left(\mathbf{x}_{w}, \mathbf{v}, t\right) v_{\perp} d \mathbf{v}=0 .
$$

Boundary conditions at the interface between the SOL and the closed flux surface region have to be added in order to couple the SOL dynamics with the core and edge physics.

Equations (3.1)-(3.3) are coupled to Maxwell equations for the evaluation of the electric and magnetic fields. We note that it might be necessary to couple the kinetic equations for the neutrals and the plasma to a calculation of radiation transport. In fact, while the plasma is typically assumed optically thin, various spectroscopic studies have pointed out that it may absorb radiation, especially at very high densities and in large machines. Photon absorptions lead to the excitation of atoms and molecules and, therefore, to an increased ionization rate - taking this process into account might be particularly important in the study of the plasma detachment.

The kinetic equations for the neutral atoms, such as (3.1), is linear in $f_{n}\left(\mathbf{x}, \mathbf{v}_{n}, t\right)$ and can be solved numerically relatively easily, despite the complexity of the collision operator. As a matter of fact, assuming the plasma dynamics is known, the solution of the Boltzmann kinetic equation can be approached by using Monte Carlo techniques. Over the years, a number of codes have been implemented to tackle the solution of the neutral kinetic equations: we mention EIRENE (Reiter et al. 1991; Schneider et al. 1992), DEGAS 2 (Stotler and Karney 1994), NIMBUS (Taroni et al. 1992), NEUT2D (Shimizu et al. 2003). These codes can include atoms and molecules, in different excited states, with the possibility of inter-species reactions (in this case the problem becomes nonlinear). The details of the interaction of the fusion fuel with the wall can be described, as well as the pumping and fueling. These codes can also be coupled to a photon transport model, which is typically treated within the numerical scheme developed for the transport of neutrals.

On the other hand, evolving the plasma dynamics involves dealing with a complex nonlinear system of equations and it is extremely challenging. The solution of the full kinetic system has been carried out by using codes based on the Particle-in-Cell method and a Monte Carlo collision operator. Simulations of this kind started in the late eighties (Chodura 1988), considering a reduced ion-to-electron mass ratio, no neutral atoms, and a very small domain. While fully three-dimensional turbulent kinetic simulations of plasma turbulence using fully kinetic codes are nowadays out of reach, present models, now run on massively parallel computers, are able to describe the interaction of multiple ion species plasmas with a large number of neutral species, impurities, and also with the plasma facing components. The most advanced codes are able to describe tens of elastic, charge-exchange, excitation, ionization and charge-exchange collision processes (Tskhakaya 2012). 
Although some two-dimensional kinetic SOL simulations have been considered (Takizuka et al. 2003), the typical setup considers a one-dimensional domain, that develops along the poloidal direction, starting at one divertor or limited plate and ending at the other. A source of plasma and heat model the cross-field transport; the particles introduced by the source flow along the magnetic field lines and are lost at the vessel walls where they are absorbed. The absorbed particles can sputter impurities, then removed from the simulations with some probability related to their cross-field transport, or be recycled as neutrals. A typical example of these simulations is described in Tskhakaya (2012). The SOL kinetic models are particularly useful to understand the role of the different impurities and neutral species present in the SOL, with the goal of deducing simplified models of the SOL dynamics. They are also used to evaluate parallel transport coefficients and the plasma boundary conditions at the vessel wall.

We finally point out that the SOL dynamics has also been approached by using gyrokinetic simulations. By ordering the gyro-motion as the fastest time scale, the three-dimensional velocity space can be reduced to a two-dimensional domain. Global gyrokinetic turbulent simulations have been extended to cover the SOL domain and take into account also neutral physics (Chang et al. 2009) as well as spatially onedimensional simulations of the tokamak SOL have been performed (Shi et al. 2014). While some advances in the study of the edge dynamics have been obtained through the use of the kinetic simulations, because of their numerical cost, fluid codes still remain the model of reference to perform the large parameter scans of SOL turbulence simulations needed to deepen our understanding of SOL physics.

\section{Fluid description of the SOL dynamics}

At the plasma edge, where collisionality plays a dominant role and kinetic effects such as particle trapping and wave-particle resonance are less important, fluid modeling is a reasonable choice to perform global turbulence simulations at a computational cost that allows wide parameter scans. Within the hypotheses that (i) the plasma distribution function is close to a local Maxwellian, (ii) the macroscopic time variations are slower than the collisional time, (iii) the scale lengths along the magnetic field are longer than the mean free path, the motion of the plasma can be described by the Braginskii momentum equation (Braginskii 1965), which for the species $\alpha=\{i, e\}$, state that

$$
m_{\alpha} n\left(\frac{\partial}{\partial t}+\mathbf{v}_{\alpha} \cdot \nabla\right) \mathbf{v}_{\alpha}=-\nabla p_{\alpha}+q_{\alpha} n\left(\mathbf{E}+\frac{1}{c} \mathbf{v}_{\perp \alpha} \times \mathbf{B}\right)-\nabla \cdot \underline{\underline{\pi_{\alpha}}}+\mathbf{R}_{\alpha}
$$

where we have assumed a quasi-neutral plasma with singly charged ions, such that $n=n_{i}=n_{e}$ and $q_{i}=-q_{e}=e$. The stress tensor is divided into a finite Larmor radius part and a viscous part and its form is evaluated by Braginskii (1965) in the limit of interest in the SOL, that is ion-cyclotron frequency larger than the collisional time, $\tau_{\text {coll }} \omega_{c i} \gg 1$. The momentum transfer vector, $\mathbf{R}_{\alpha}$, can be neglected for ions, while it has to be kept for electrons and is equal to $\mathbf{R}_{e}=n \mathbf{b}\left[e j_{\|} / \sigma_{\|}-0.71 \nabla_{\|} T_{e}\right]$, with $\sigma_{\|}=1.96 n e^{2} \tau_{e} / m_{e}$ being the parallel conductivity and $j_{\|}=e n\left(V_{\| i}-V_{\| e}\right)$. The friction in the direction perpendicular to the magnetic field can also be neglected.

While there exist some attempts to solve the full set of Braginskii equations to study the plasma dynamics in the SOL (Knight et al. 2012), typical simulations adopt the drift ordering, which is based on assuming $\partial_{t} \ll \omega_{c i}$ and perpendicular scale lengths longer than the ion gyroradius, $\rho_{i} \nabla_{\perp} \ll 1$ (see e.g., Zeiler et al. (1997) for a 
detailed discussion). Within the drift ordering, it is useful to split the analysis of the plasma motion into the direction parallel and perpendicular to the magnetic field, by decomposing $\mathbf{V}_{\alpha}=V_{\| \alpha} \mathbf{b}+\mathbf{v}_{\perp \alpha}$. By assuming that the viscous terms are small, the drift ordering indicates that the leading order term for $\mathbf{v}_{\perp}$ is $\mathbf{v}_{\perp \alpha 0}=\mathbf{v}_{E}+\mathbf{v}_{d \alpha}$, where $\mathbf{v}_{E}=c \mathbf{E} \times \mathbf{B} / B^{2}$ is the $\mathbf{E} \times \mathbf{B}$ drift and $\mathbf{v}_{d \alpha}=c \mathbf{b} \times \nabla p_{\alpha} /\left(m_{\alpha} n_{\alpha} \omega_{c \alpha}\right)$ is the diamagnetic velocity. The higher-order term in the velocity is the polarization drift, which can be expressed as

$$
\mathbf{v}_{\mathrm{pol}, \alpha}=\mathbf{v}_{\perp \alpha}-\mathbf{v}_{\perp \alpha 0} \simeq \frac{\mathbf{b}}{\omega_{c \alpha}} \times \frac{\mathrm{d}}{\mathrm{d} t} \mathbf{v}_{\perp \alpha 0}+\frac{1}{n m_{\alpha} \omega_{c \alpha}}\left[\mathbf{b} \times\left(\nabla \cdot \underline{\underline{\pi_{\alpha}}}-\mathbf{R}_{\alpha}\right)\right],
$$

where $d_{t}=\partial_{t}+\left(\mathbf{v}_{E}+\mathbf{v}_{d \alpha}+V_{\| \alpha} \mathbf{b}\right) \cdot \nabla$ because, according to the ordering we are considering, $\mathbf{v}_{\text {pol }}$ can be dropped.

The drift-reduced Braginskii equations that are typically considered to study the plasma dynamics in the SOL can now be derived. For the perpendicular motion of electrons we can set $\mathbf{v}_{\mathrm{pol}, e}=0$, since electron finite Larmor radius effects are negligible and the viscous part of the electron pressure tensor is proportional to $m_{e} / m_{i}$. Therefore, the electron continuity equation reads

$$
\frac{\partial n}{\partial t}+\nabla \cdot\left[n\left(\mathbf{v}_{E}+\mathbf{v}_{d e}+V_{\| e} \mathbf{b}\right)\right]=S
$$

where $S$ is the plasma density source. By subtracting the ion and electron continuity equations, because of quasi neutrality, one obtains

$$
\nabla \cdot\left(n \mathbf{v}_{\mathrm{pol}, i}\right)+\nabla_{\|} \cdot\left(j_{\|} \mathbf{b}\right) / e+\nabla \cdot\left[n\left(\mathbf{v}_{d i}-\mathbf{v}_{d e}\right)\right]=0,
$$

which is equivalent to $\nabla \cdot \mathbf{j}=0$, meaning that the displacement current is negligible and that there is no charge accumulation in the plasma. Equation (4.4) is usually called the vorticity equation, because the development of $\nabla \cdot\left(n \mathbf{v}_{\text {pol, } i}\right)$ leads to a term proportional to $\partial_{t} \nabla_{\perp}^{2} \phi$, with the term $\nabla_{\perp}^{2} \phi$ representing the vorticity function of a fluid moving with the $\mathbf{v}_{E}$ velocity.

Maxwell's equations for the electric and magnetic field, $\mathbf{E}$ and $\mathbf{B}$, can be significantly simplified. As $\beta=8 \pi\left(p_{e}+p_{i}\right) / B^{2} \ll 1$, the current flowing in the SOL is small and the variation of the parallel magnetic field negligible. The perturbed magnetic field, $\delta \mathbf{B}$, is, therefore, perpendicular to the equilibrium one, and given by:

$$
\delta \mathbf{B}=-\nabla \times\left(\psi \mathbf{b}_{0}\right) \simeq \mathbf{b}_{0} \times \nabla_{\perp} \psi=\delta \mathbf{B}_{\perp}
$$

where $\mathbf{b}_{0}$ is the unit vector in the direction of the unperturbed magnetic field and the perturbed potential vector, parallel to the background magnetic field, $\delta \mathbf{A}=-\psi \mathbf{b}_{0}$, is given by Ampère law:

$$
\nabla_{\perp}^{2} \psi=\frac{4 \pi}{c} j_{\|}
$$

The perpendicular component of $\delta \mathbf{A}$ can be neglected because the spatial scales in the parallel direction are much larger than the ones in the perpendicular direction. The projection of the electric field along the parallel direction can be expressed as

$$
E_{\|}=-(\mathbf{b} \cdot \nabla) \phi+\frac{1}{c} \frac{\partial \psi}{\partial t}
$$


By projecting the electron momentum equation, (4.1), along the parallel direction, the equation for the parallel electron velocity, $V_{\| e}$ is obtained:

$$
\begin{aligned}
m_{e} n \frac{\partial V_{\| e}}{\partial t}+\frac{e n}{c} \frac{\partial \psi}{\partial t}= & -\frac{c m_{e} n \mathbf{b}}{B} \cdot\left(\nabla \phi \times \nabla V_{\| e}\right)-m_{e} n V_{\| e}(\mathbf{b} \cdot \nabla) V_{\| e}-\frac{2}{3}(\mathbf{b} \cdot \nabla) G_{e} \\
& -\frac{e^{2} n^{2}}{\sigma_{\|}}\left(V_{\| e}-V_{\| i}\right)+e n(\mathbf{b} \cdot \nabla) \phi-T_{e}(\mathbf{b} \cdot \nabla) n \\
& -1.71 n(\mathbf{b} \cdot \nabla) T_{e}
\end{aligned}
$$

where $G_{e}$, is a function related to the finite Larmor radius part of the stress tensor (Ricci et al. 2012). The ion momentum equation is obtained by summing the ion and electron parallel momentum equations and neglecting the electron inertia. One obtains:

$$
\begin{aligned}
m_{i} n \frac{\partial V_{\| i}}{\partial t}= & -\frac{c m_{i} n \mathbf{b}}{B} \cdot\left(\nabla \phi \times \nabla V_{\| i}\right)-m_{i} n V_{\| i}(\mathbf{b} \cdot \nabla) V_{\| i}-\frac{2}{3}(\mathbf{b} \cdot \nabla) G_{i} \\
& -n(\mathbf{b} \cdot \nabla) T_{e}-T_{e}(\mathbf{b} \cdot \nabla) n
\end{aligned}
$$

Equations (4.3), (4.4), and (4.6)-(4.9), completed by Braginskii equations for the electron and ion temperature, and an appropriate set of boundary conditions (Loizu et al. 2012), constitute the drift-reduced model that has been considered to simulate plasma turbulence in the SOL. In the past few years a number of codes have been developed for solving these equations, or a simplified versions. We mention the codes BOUT++ (Dudson et al. 2009), GBS (Ricci et al. 2012), and TOKAM3X (Tamain et al. 2010). Thanks to their simulations, significant progress has been recently made in the understanding of the physics underlying SOL turbulence. We mention the identification of the saturation mechanism of plasma turbulence in the SOL (Ricci and Rogers 2013) and of the SOL turbulent regimes (Mosetto et al. 2013). The role of electromagnetic fluctuations has been pointed out (Halpern et al. 2013b). The scaling of the SOL width (Halpern et al. 2014), the origin of intrinsic toroidal rotation (Loizu et al. 2014), the value of the SOL electrostatic potential (Loizu et al. 2013) are other recent achievements. A number of comparisons with experimental devices has been performed (Halpern et al. 2013a).

By taking into account the fact that turbulence in the SOL is dominated by modes that are strongly elongated in the direction parallel to the magnetic field line, one can simplify the drift-reduced equations to a two-dimensional model that evolve the plasma dynamics on a plane perpendicular to the magnetic field. The two-dimensional modeling has been mostly applied to study the propagation of blobs (D'Ippolito et al. 2011), plasma structures of enhanced density and temperature that can give a significant transient load on the vessel walls. The results of the two-dimensional modeling have been compared with experimental measurements, showing fairly good agreement with respect to the turbulent properties (Garcia et al. 2006; Russell et al. 2011). Among the codes mostly employed in two-dimensional SOL modeling, we cite TOKAM2D (Sarazin and Ghendrih 1998), ESEL (Naulin et al. 1998), and SOLT (D'Ippolito and Myra 2003).

We remark that, while most of the SOL simulations are based on the drift-reduced Bragiskii equations, more advanced models have been considered that, for example, take into account finite gyromotion effects (Ribeiro and Scott 2005). Closures of the fluid equations that might be also valid in low-collisionality regimes have been considered deriving those from kinetic models (Omotani and Dudson 2013). The fluid simulations are typically considered in the low-recycling regime, neglecting the 
interaction with the neutrals. However, recent progress has been carried out to couple the drift-reduced Braginskii equations with a model for the neutral atoms (Mekkaoui et al. 2014; Marandet et al. 2013).

\section{Phenomenological description}

Despite the large progress that has been performed in first-principle simulations of SOL dynamics, to date, tokamak divertors and wall systems are still designed largely on the basis of empirical extrapolation of SOL plasma and neutral dynamics observed in present devices, supported by basic theoretical considerations. As empirical codes, even fully validated against the measurements taken in existing devices, cannot reliably predict the SOL dynamics in conditions far from existing experience, we do not expect detailed quantitative predictions, rather the identification of the trends required for extrapolations. A fairly recent review of the status on SOL empirical modeling is contained in Loarte et al. (2007).

In their simplest form, the codes solve a reduced set of Braginskii fluid equations for $n, T_{i}, T_{e}$, and $V_{\| i}$ (quasi neutrality and ambipolar flow are assumed), but they can also incorporate a multi-species fluid description. Transport across the magnetic field lines is described through an ad hoc diffusion-advection process. Parallel transport is modeled using classical heat conductivity with flux limit factors, to mimic kinetic effects. When run in interpretative manner, the free diffusive and convective parameters are adjusted to fit the experimental data. These fits are then used to evaluate the SOL profiles when the codes are run in predictive mode. In recent years, the description of the plasma dynamics was improved by inclusion of stationary $\mathbf{E} \times \mathbf{B}$ and $\nabla B$ drifts and currents. The plasma description is coupled to a MonteCarlo model for neutral particles, such as the ones mentioned in Sec. 3. The latter is essential to describe pumping, fueling, and detailed plasma-material interactions.

Among the empirical SOL modeling codes, we mention SOLPS (formerly B2Eirene) (Reiter et al. 1991; Schneider et al. 1992), EDGE2D (Simonini et al. 1994), UEDGE (Rognlien et al. 1994), and SOLDOR (Shimizu et al. 2003). These codes are widely used both in interpretative and in predictive modes. They represent a considerable investment of the fusion community. For example, the workhorse SOL simulation code used for the ITER divertor design (Pitts et al. 2009), SOLPS, consists in about 200000 lines of source codes, with over 100 professional-years invested in its development (Kukushkin et al. 2011).

There are phenomena that are not implemented in major codes, such as SOLPS, because of the very different time scales of interest or because it is possible to decouple their dynamics from the one of the main plasma. Among those we mention the propagation of fast ions leaving the core plasma, which is addressed for example by ASCOT (Heikkinen et al. 2001), a kinetic Monte-Carlo code that follows the fast ions as they outflow from the pedestal, interact with the SOL plasma and neutrals (whose profile is evaluated by the major codes), and are lost at the divertor plates. Fast ion flux to the divertor plates can be responsible for sharp peaks on the divertor power loads in some JET discharges. Strong pulse of heat and particles, caused by edge localized modes (ELMs) or due to disruptions are modeled by using numerical codes such as HEIGHTS (Hassanein and Konkashbaev 2003) and FOREV-2 (Pestchanyi et al. 2002). They are able to describe the effect of the strong pulse on the target plates, evaluating the erosion of the materials and the transport of radiation. Finally, impurity dynamics is treated with codes such as REDEP (Brooks et al. 2003) and ERO-JET (Kirschner et al. 2003) (for carbon) and DIVIMP (Geier et al. 2003) (for 
tungsten). Those codes, based on the Monte Carlo method, are able to model the sputtering of the impurities from the surfaces and their transport in the edge. Because of its difficulty, there has been a smaller effort in evaluating the impurity transport from the SOL into the core.

The modeling of the SOL has pointed out that the parameters that play the major role in determining the SOL profiles are: the power entering the SOL, the pressure of the fusion fuel, the pumping speed, the fusion power through the concentration of helium, the ratio between gas puffing and core fueling, and the connection length of the magnetic field (Loarte et al. 2007). The results of the modeling activities have had an impact on the ITER divertor design (Kukushkin et al. 2011). For example, SOLPS simulations showed that partial detachment is facilitated by a V-shaped intersection of the target plates with the divertor floor. Also, in order to reduce the load imbalance between the outer and inner divertor legs, the structures supporting the divertor dome have been realized to allow high gas conductance.

\section{Conclusions}

Because of the key role of the SOL in determining the performances of a fusion device, understanding its physics is of the utmost importance for the success of the entire fusion program. As a matter of fact, some of the most crucial issues that fusion is facing today are related to the plasma dynamics in the SOL region. While phenomenological codes have been used as a design tool for ITER and still remain the tools of reference, the need to support their use with the results of first-principles simulations is increasingly felt by the community in view of the design of future fusion devices.

In the last few years, significant progress has been made in the first-principles approach to the SOL dynamics and a number of complimentary models have been considered. Most likely, in the next few years, fluid models, such as the ones based on the drift-reduced Braginskii equations, will remain the workhorse of SOL firstprinciples simulations. At the same time, fluid models can benefit from the results of SOL kinetic simulations that are currently carried out in a reduced geometry. In fact, the kinetic simulations point out the most important processes involving the neutral atoms that fluid codes have to take into account. Kinetic codes also provide the fluid models with the necessary boundary conditions and fluid closures. The development of more complex codes based on the gyrokinetic model already started and it is likely that, with the advancement of the computational capabilities, they will become the future tool of reference. At the same time, progress is urgently needed in the development of a model that allows the coupling of the SOL physics with the plasma dynamics in the closed flux surface region. The description of the interplay between these two regions is believed necessary for the understanding of the low to high confinement transition and the ELM physics.

\section{Acknowledgements}

Useful discussions with A. Fasoli, I. Furno, F. Halpern, J. Loizu, F. Riva and C. Wersal are acknowledged. The work, carried out within the framework of the EUROfusion Consortium, was supported by the Swiss National Science Foundation and by the European Union Horizon 2020. The views and opinions expressed herein do not necessarily reflect those of the European Commission. 


\section{REFERENCES}

Braginskii, S. I. 1965 The plasma boundary of magnetic fusion devices. In: Reviews of Plasma Physics, Vol. 1, New York: Consultants Bureau, p. 205.

Brooks, J. N., Kirschner, A., Whyte, D. G., Ruzic, D. N. and Alman, D. A. 2003 Advances in the modeling of chemical erosion/redeposition of carbon divertors and application to the JET tritium codeposition problem. J. Nuclear Mater. 313-316(0), 424-428.

Chang, C. S. et al. 2009 Whole-volume integrated gyrokinetic simulation of plasma turbulence in realistic diverted-tokamak geometry. J. Phys.: Conf. Ser. 180(1), 012057.

Chodura, R. 1988 Nonlocal heat-flux in the scrape-off layer of a high-temperature plasma. Contrib. Plasma Phys. 28(4-5), 325-327.

D’Ippolito, D. A., Myra, J. R. and Zweben, S. J. 2011 Convective transport by intermittent blobfilaments: comparison of theory and experiment. Phys. Plasmas (1994-present) 18(6), 060501.

Dudson, B. D., Umansky, M. V., Xu, X. Q., Snyder, P. B. and Wilson, H. R. 2009 BOUT++: a framework for parallel plasma fluid simulations. Comput. Phys. Commun. 180(9), 1467-1480.

D'Ippolito, D. A. and Myra, J. R. 2003 Blob stability and transport in the scrape-off-layer. Phys. Plasmas (1994-present) 10(10), 4029-4039.

Eich, T., Sieglin, B., Scarabosio, A., Fundamenski, W., Goldston, R. J. and Herrmann, A. 2011 InterELM power decay length for JET and ASDEX Upgrade: measurement and comparison with heuristic drift-based model. Phys. Rev. Lett. 107, 215001.

Garcia, O. E., Horacek, J., Pitts, R. A., Nielsen, A. H., Fundamenski, W., Graves, J. P., Naulin, V. and Rasmussen, J. J. 2006 Interchange turbulence in the TCV scrape-off layer. Plasma Phys. Control. Fusion 48(1), L1.

Geier, A., Krieger, K., Elder, J. D., Pugno, R., Rohde, V. and Team, The ASDEX Upgrade 2003 Modeling of tungsten transport in the SOL for sources at the central column of ASDEX upgrade using DIVIMP. J. Nucl. Mater. 313-316(0), 1216-1220.

Halpern, F. D., Ricci, P., Jolliet, S., Loizu, J. and Mosetto, A. 2014 Theory of the scrape-off layer width in inner-wall limited tokamak plasmas. Nucl. Fusion 54(4), 043003.

Halpern, F. D. et al. and Contributors, JET-EFDA 2013a Theory-based scaling of the SOL width in circular limited tokamak plasmas. Nucl. Fusion 53(12), 122001.

Halpern, F. D., Jolliet, S., Loizu, J., Mosetto, A. and Ricci, P. 2013b Ideal ballooning modes in the tokamak scrape-off layer. Phys. Plasmas (1994-present) 20(5), 052306.

Hassanein, A. and Konkashbaev, I. 2003 Comprehensive modeling of ELMs and their effect on plasma-facing surfaces during normal tokamak operation. J. Nucl. Mater. 313-316(0), 664669. (plasma-Surface Interactions in Controlled Fusion Devices 15).

Heikkinen, J. A., Kiviniemi, T. P., Kurki-Suonio, T., Peeters, A. G. and Sipil, S. K. 2001 Particle simulation of the neoclassical plasmas. J. Comput. Phys. 173(2), 527-548.

ITER Physics Expert Group on Divertor, on Divertor Modelling, ITER Physics Expert Group, Database \& Editors, ITER Physics Basis 1999 Chapter 4: Power and particle control. Nucl. Fusion 39(12), 2391.

Kirschner, A., Brooks, J. N., Philipps, V., Coad, J. P. and contributors to the EFDAJET Workprogramme 2003 Hydrocarbon transport in the mkiia divertor of JET. Plasma Phys. Control. Fusion 45(3), 309.

Knight, P. J., Thyagaraja, A., Edwards, T. D., Hein, J., Romanelli, M. and McClements, K. G. 2012 Centori: a global toroidal electromagnetic two-fluid plasma turbulence code. Comput. Phys. Commun. 183(11), 2346-2363.

Kukushkin, A. S., Pacher, H. D., Kotov, V., Pacher, G. W. and Reiter, D. 2011 Finalizing the \{ITER\} divertor design: the key role of SOLPS modeling. Fusion Eng. Des. 86(12), 2865-2873.

LaBombard, B., Golfinopoulos, T., Brunner, D., Terry, J. L., Davis, E., Greenwald, M., Hughes, J. W. and Team, Alcator C-Mod 2014 New insights on boundary plasma turbulence and the quasi-coherent mode in Alcator C-Mod using a Mirror Langmuir Probe. Phys. Plasmas 21(5), 056108.

Loarte, A. et al., Divertor Physics Topical 2007 Chapter 4: Power and particle control. Nucl. Fusion 47(6), S203.

Loizu, J., Ricci, P., Halpern, F. D. and Jolliet, S. 2012 Boundary conditions for plasma fluid models at the magnetic presheath entrance. Phys. Plasmas (1994-present) 19(12), 122307. 
Loizu, J., Ricci, P., Halpern, F. D., Jolliet, S. and Mosetto, A. 2013 On the electrostatic potential in the scrape-off layer of magnetic confinement devices. Plasma Phys. Control. Fusion 55(12), 124019.

Loizu, J., Ricci, P., Halpern, F. D., Jolliet, S. and Mosetto, A. 2014 Intrinsic toroidal rotation in the scrape-off layer of tokamaks. Phys. Plasmas (1994-present) 21(6), 062309.

Marandet, Y., Tamain, P., Futtersack, R., Ghendrih, Ph., Bufferand, H., Genesio, P. and Mekkaoui, A. July 2013 Influence of neutral particles on scrape-off layer turbulence with application to the interpretation of fast camera data. Journal of Nuclear Materials Volume 438, Supplement, Pages S518-S521.

Mekkaoui, S., Dudson, D., Reiter, D., Kotov, V. and Boerner, P. 2014 Self-consistent turbulencerecycling modeling in the lapd device. In: Proc. 21st Int. Conf. on Plasma Surface Interaction in Controlled Fusion Devices, Kanazawa, Japan.

Mosetto, A., Halpern, F. D., Jolliet, S., Loizu, J. and Ricci, P. 2013 Turbulent regimes in the tokamak scrape-off layer. Phys. Plasmas (1994-present) 20(9), 092308.

Naulin, V., Nycander, J. and Rasmussen, J. J. 1998 Equipartition and transport in two-dimensional electrostatic turbulence. Phys. Rev. Lett. 81, 4148-4151.

Omotani, J. T. and Dudson, B. D. 2013 Non-local approach to kinetic effects on parallel transport in fluid models of the scrape-off layer. Plasma Phys. Control. Fusion 55(5), 055009.

Pestchanyi, S. E., Wrz, H. and Landman, I. S. 2002 Impurity production and edge plasma pollution during iter-feat elms. Plasma Phys. Control. Fusion 44(6), 845.

Pitts, R. A., Kukushkin, A., Loarte, A., Martin, A., Merola, M., Kessel, C. E., Komarov, V. and Shimada, M. 2009 Status and physics basis of the iter divertor. Phys. Scr. 2009(T138), 014001.

Reiter, D., Kever, H., Wolf, G. H., Baelmans, M., Behrisch, R. and Schneider, R. 1991 Helium removal from tokamaks. Plasma Phys. Control. Fusion 33(13), 1579.

Ribeiro, T. T. and Scott, B. 2005 Tokamak turbulence computations on closed and open magnetic flux surfaces. Plasma Phys. Control. Fus. 47(10), 1657.

Ricci, P., Halpern, F. D., Jolliet, S., Loizu, J., Mosetto, A., Fasoli, A., Furno, I. and Theiler, C. 2012 Simulation of plasma turbulence in scrape-off layer conditions: the GBS code, simulation results and code validation. Plasma Phys. Control. Fusion 54(12), 124047.

Ricci, P. and Rogers, B. N. 2013 Plasma turbulence in the scrape-off layer of tokamak devices. Phys. Plasmas (1994-present) 20(1), 010702.

Rognlien, T. D., Brown, P. N., Campbell, R. B., Kaiser, T. B., Knoll, D. A., Mchugh, P. R., Porter, G. D., Rensink, M. E. and Smith, G. R. 1994 2-d fluid transport simulations of gaseous radiative divertors. Contrib. Plasma Phys. 34(2-3), 362-367.

Russell, D. A., Myra, J. R., D’Ippolito, D. A., Munsat, T. L., Sechrest, Y., Maqueda, R. J., Stotler, D. P., Zweben, S. J. and Team, The NSTX 2011 Comparison of scrape-off layer turbulence simulations with experiments using a synthetic gas puff imaging diagnostic. Phys. Plasmas (1994-present) 18(2), 022306.

Sarazin, Y. and Ghendrih, Ph. 1998 Intermittent particle transport in two-dimensional edge turbulence. Phys. Plasmas (1994-present) 5(12), 4214-4228.

Schneider, R., Reiter, D., Zehrfeld, H. P., Braams, B., Baelmans, M., Geiger, J., Kastelewicz, H., Neuhauser, J. and Wunderlich, R. 1992 B2-EIRENE simulation of ASDEX and ASDEXUpgrade scrape-off layer plasmas. Proc. 10th Int. Conf. on Plasma-Surface Interactions in Controlled Fusion Devices, J. Nucl. Mater. 196-198(0), 810-815.

Shi, E. L., Hakim, A. H. and Hammett, G. W. 2014 A gyrokinetic 1D scrape-off layer model of an ELM heat pulse. arXiv:1409.2520.

Shimizu, K., Takizuka, T., Sakurai, S., Tamai, H., Takenaga, H., Kubo, H. and Miura, Y. 2003 Simulation of divertor detachment characteristics in JT-60 with superconducting coils. $J$. Nucl. Mater. 313-316(0), 1277-1281.

Simonini, R., Corrigan, G., Radford, G., Spence, J. and Taroni, A. 1994 Models and numerics in the multi-fluid 2-d edge plasma code EDGE2D/U. Contrib. Plasma Phys. 34(2-3), 368-373.

Stangeby, P. C. 2000 The Plasma Boundary of Magnetic Fusion Devices. Bristol and Philadelphia: Institute of Physics Publishing.

Stotler, D. and Karney, C. 1994 Neutral gas-transport modeling with DEGAS-2. Contrib. Plasma Phys. 34(2-3), 392-397.

Takizuka, T., Hosokawa, M. and Shimizu, K. 2003 Two-dimensional particle simulation of the flow control in SOL and divertor plasmas. J. Nucl. Mater. 313-316(0), 1331-1334. 
Tamain, P., Ghendrih, Ph., Tsitrone, E., Grandgirard, V., Garbet, X., Sarazin, Y., Serre, E., Ciraolo, G. and Chiavassa, G. 2010 Tokam-3d: a 3d fluid code for transport and turbulence in the edge plasma of tokamaks. J. Comput. Phys. 229(2), 361-378.

Taroni, A., Corrigan, G., Radford, G., Simonini, R., Spence, J. and Weber, S. 1992 The multi-fluid codes EDGEID and EDGE2D: models and results. Contrib. Plasma Phys. 32(3-4), 438-443.

Tskhakaya, D. 2012 On recent massively parallelized PIC simulations of the SOL. Contrib. Plasma Phys. 52(5-6), 490-499.

Yagi, M., Itoh, S.-I., Itoh, K. and Diamond, P. H. 2008 Disparate scale nonlinear interactions in edge turbulence. Contrib. Plasma Phys. 48(1-3), 13-22.

Zeiler, A., Drake, J. F. and Rogers, B. 1997 Nonlinear reduced Braginskii equations with ion thermal dynamics in toroidal plasma. Phys. Plasmas (1994-present) 4(6), 2134-2138.

Zohm, H. et al. 2013 On the physics guidelines for a tokamak DEMO. Nucl. Fusion 53(7), 073019. 